\title{
Laparoscopy is the Gold Standard in Ovarian Pathologies in Childhood: Clinical Evaluation and Literature Review
}

\author{
Gülnur GÖLLÜ1, Ufuk ATEŞ¹, Nil YAŞAM TAŞTEKİN', Ergun ERGÜN¹, Meltem BİNGÖL KOLOĞLU1 \\ Aydın YAĞMURLU' ${ }^{1}$ Tanju AKTUĞ ${ }^{1}$, Hüseyin DINDAR' ${ }^{1}$, Ahmet Murat ÇAKMAK \\ Ankara, Turkey
}

\begin{abstract}
OBJECTIVE: Ovarian cysts and related torsions are the most common adnexial pathologies in childhood. Diagnostic laparoscopy is the gold standard approach for differential diagnosis. It is aimed to evaluate the data of patients who had surgery for ovarian pathology.

STUDY DESIGN: Fifty-three girls of ten years are included the study. Demographic data, medical history, physical examination, laboratory and radiologic investigations, surgical procedure, histopathology, complications and follow up periods are recorded.

RESULTS: The median age of children was 13. Excluding the patients who were diagnosed in intrauterine period $(n=4)$, all of the patients had abdominal pain $(92 \%)$. Laparoscopic cystectomy $(56.7 \%)$, detorsion and cystectomy $(16.9 \%)$ and oopherectomy $(16.9 \%)$ were performed in patients with cysts. Diagnostic laparoscopy $(3,8 \%)$ and laparoscopic detorsion $(5.7 \%)$ were performed in patients without cyst. Dermoid cysts were found in three children and teratomas in two. Patients were discharged in postoperative second day.
\end{abstract}

CONCLUSION: Diagnostic laparoscopy is the gold standard approach for differential diagnosis. Laparoscopic approach should be chosen because of shorter hospital stay, shorter healing period, and better cosmetic results. Besides, less pelvic adhesions compared to laparotomy in laparoscopy is important for further fertility.

Keywords: Children, Cyst, Laparoscopy, Ovary, Torsion

Gynecol Obstet Reprod Med 2016;22(3):144-148ＤOI: 10.201613/GORM.2016.626

\section{Introduction}

Ovarian cysts and related torsions are the most common adnexial pathologies in childhood (1-3). Children with ovarian cysts can present with hormonal imbalance, cyst rupture, hemorrhage and torsion (4-6) Ovarian torsion is an emergency; it can be with or without a cyst. Besides, benign or malignant neoplasm can be found (2). Common complaint is pelvic or abdominal pain $(4,7)$. Although ultrasonography and color Doppler ultrasonography may be useful for diagnosis $(2,8,9)$, diagnostic laparoscopy is the gold standard approach for differential diagnosis (1). It is aimed to evaluate the data of patients who had surgery due to ovarian pathology in past ten years.

${ }^{1}$ Ankara University School of Medicine Department of Pediatric Surgery, Address of Correspondence: Gülnur Göllü

Ankara University School of Medicine Department of Pediatric Surgery, 06100, Dikimevi, Ankara, Turkey

drggollu@yahoo.com

Submitted for Publication

05.08 .2016

Accepted for Publication:
06.09 .2016

\section{Material and Method}

The study was performed in adherence to the Declaration of Helsinki. A written informed consent was signed by the legal guardians of each child. The charts of patients with ovarian pathologies between January 2006 and May 2016 were retrospectively examined. Fifty-three girls were included for the study. Demographic data, medical history, physical examination, laboratory and radiologic investigations, complications, surgical procedure, histopathological evaluation and follow up periods were recorded.

Ultrasonography and color Doppler ultrasonography were performed to all children for diagnosis. Increase in diameter of ovary and cyst, peripherally located folliculles or solid component of cysts were evaluated via ultrasonography. Blood flow was tested via color Doppler ultrasonography. The children were evaluated with medical history, physical examination, blood tests and radiologic imaging. Children with pain complaints for more than 48 hours and children in whom torsion cannot be excluded underwent surgery emergently. Children who had persistent cysts more than five $\mathrm{cm}$ diameter after one or two menstrual cycles and children who had cysts 
with solid components underwent elective surgery. Children were located in supine position on the table. Nasogastric tube was placed to all children before the anesthesia by the aim of gastric decompression. Bladder was emptied before the procedure. All the patients were intubated. Umbilical 5-10 mm incision was made to place camera trocar with open technique. In children less than $10 \mathrm{~kg} ; 3 \mathrm{~mm}$ working trocars were placed bilaterally in upper lateral side of rectus abdominis muscle and in children more than $10 \mathrm{~kg}, 5 \mathrm{~mm}$ trocars were placed bilaterally in lower lateral side of rectus abdominis muscle. If the cyst was so big that it crossed the umbilical line, camera trocar was placed in epigastrium and the working ports in upper quadrants. The table was in 30 degree Trendelenburg position and the monitor was placed caudally. Surgeon and cameraman positioned contralaterally to the lesion.

All the torsioned ovaries were detorsioned. The cysts were aspirated with a Veress or injector needle percutaneously. Cystic wall and fluid was extracted for histopathological examination. The cystectomy was performed with hook cautery or vascular sealing devices and the ovarian tissue was preserved. If the cyst was too big to remove from the umbilical trocar the cyst was aspirated percutaneously and removed from the umbilicus and laparoscopy assisted procedure was performed. Solid masses were removed via endobag through the umbilicus. Nasogastric tube was removed immediately after the surgery and the patients were fed postoperatively at sixth hour. Tumor marker tests were performed in children who had complicated cyst and solid masses. If the ovarian pathology was hormonally active, endocrinology consultation was requested.

\section{Results}

Fifty-three girls of ten years were included the study. The ages of children varied between two days and eighteen years (median thirteen years). Four babies had prenatal diagnosis. Excluding the patients who were diagnosed in intrauterine period $(n=4)$, all of the patients had abdominal pain $(n=49$, $92 \%)$. Seven children had recurrent or long-term abdominal pain $(13 \%)$. Nausea and vomiting were accompanying to abdominal pain in 15 children $(28 \%)$. Palpable mass was recorded in six children $(11 \%)$. Leukocytosis $(>10 \times 109 / \mathrm{L})$ was found in nineteen children (35.8\%). Tumor markers were evaluated in 13 patients, and no pathology was revealed. All of the patients had ultrasonography and color Doppler ultrasonography and four children had computed tomography imaging. Right ovarian pathology was revealed in 28 of the patients $(52.8 \%)$, left in $23(43.3 \%)$ and bilateral in two $(3.7 \%)$ (Table 1). All procedures started laparoscopically. Cystectomy was performed in 30 children (56.7\%), cystectomy and detorsion in nine $(16.9 \%)$, oopherectomy in nine $(16.9 \%)$, only detorsion in three $(5.7 \%)$, and no pathology was detected in two (3.8\%) (Table 2). Mini- laparotomy and oopherectomy was performed in a newborn with an ovarian cyst with calcifications and solid components.
Mean cyst diameter was $5.94 \mathrm{~cm}(1.5-19 \mathrm{~cm})$. Two of them were giant cysts with a diameter of more than $15 \mathrm{~cm}$. Histopathologic examination of contents revealed simple cysts in 22 children and hemorrhagic cysts in 19. Dermoid cysts were found in three children and teratomas in two. In four children, gross necrosis in ovary was found. No malignant cyst was found.

Ultrasonography was concordant with diagnostic laparoscopy in all of the children with cysts. In 14 torsion cases, ultrasonography revealed increase in size in eight children, cysts in two children and peripheral follicules in one child. Color Doppler ultrasonography showed no blood flow in four children $(28.5 \%)$. In one child, torsion was seen in laparoscopy even though ultrasonography and color Doppler ultrasonography showed no signs of torsion. In one child with increase in size and one with no blood flow in color Doppler ultrasonography, laparoscopy was performed but no pathology was identified in laparoscopy.

Only in one child laparoscopic approach revealed suboptimal surgical access and laparotomy was performed, consequently. Children were discharged postoperative 1-7th days (mean 2 days). No other complications were seen. In follow up period, one child presented with ipsilateral ovarian cyst $(>5$ $\mathrm{cm}$ diameter) and underwent cystectomy.

Table 1: Demographic data, physical examination, laboratory values and pathological site of ovary

\begin{tabular}{lc}
\hline Age & 2 days-18 years \\
\hline Abdominal pain & $49(92 \%)$ \\
Nausea- vomiting & $15(28 \%)$ \\
Palpable mass & $6(11 \%)$ \\
Leukocytosis (>10x109/L) & $19(35.8 \%)$ \\
Pathologic site & \\
$\quad$ Right & $28(52.83 \%)$ \\
$\quad$ Left & $23(43.3 \%)$ \\
$\quad$ Bilateral & $2(3.77 \%)$ \\
\hline
\end{tabular}

Table 2: Laparoscopic procedure in ovarian pathologies

\begin{tabular}{ll}
\hline Laparoscopic procedures & \\
Cystectomy & $30(56.7 \%)$ \\
Cystectomy+ Detorsion & $9(16.9 \%)$ \\
Oophrectomy & $9(16.9 \%)$ \\
Detorsion & $3(5.7 \%)$ \\
Diagnostic laparoscopy & $2(3.8 \%)$
\end{tabular}

\section{Discussion}

The data of the girls who applied to our clinics with an ovarian pathology in ten years, were collected. The abdominal pain was determined to be an important clinical factor in ovarian pathologies. Although radiological imaging was useful in diagnosing a cyst or a mass, it can give false results if torsion 
was present. Minimal invasive surgery seems superior for diagnosis and treatment.

Functional ovarian cysts are follicule and corpus luteum cysts and they make up $45 \%$ of all adnexial pathologies (6). It can be seen in fetal - neonatal period with hormone stimulation or in the perimenarchal period with dysfunctional ovulation (6). Patients with ovarian cysts can present to emergency with rupture of the cyst, hemorrhage or torsion (4-6). In fact, ovarian cyst ruptures and hemorrhages can be seen in ovarian cycle as a physiological state. Middle pain (Mittelschmerz) can be seen with follicule rupture during ovulation and peritoneal free fluid can cause pain. Hemorrhagic cyst is caused by the stretching of the ovarian capsule and the rupture of the cyst causing peritoneal irritation. It cannot be determined which cyst is pathological or physiological or caused by the hemorrhage of the cyst. However in adults, cysts, which have a diameter below $25 \mathrm{~mm}$, are called a follicule and the bigger ones are called cysts. Physiological cysts can be specified as serous or musinous cystic adenomas (4). They can present to emergency with an acute onset of abdominal pain, nausea, and vomiting. Adults, experienced in Mittelschmerz, know this pain and they can guess it will end in 48 hours. They can be followed up with simple analgesics (4). However kids are not familiar to this pain, because of this, differential diagnosis must be evaluated. In ultrasonography imaging, hemorrhagic cysts are reported as ovarian cyst with typical mixed, often bizarre, internal echoes suggestive of blood cloud. But in the setting of ovarian cyst rupture, normal ovaries but site specific tenderness and free fluid in the pouch of Douglas is reported (4).

Hemorrhagic cysts must be followed up closely and are expected to resolve in six weeks. Laparoscopic indications are defined as, hemodynamic compromise, diagnostic uncertainty or likelihood of torsion, no relief of symptoms within 48 hours of presentation, increasing hemoperitoneum on ultrasonography or falling hemoglobin concentration $(2,4,6,7,10)$ Cystic teratomas form the biggest group in benign lesions (7). Malign lesions are seen as much as $1.1 \% .7$ Templeman et al. reported $57.9 \%$ of the adnexial masses are in concordance with cysts. Other masses are reported as neoplasms but $75 \%$ of these are reported benign (11). In our study, in 41 of the patients (77.3\%) cyst was determined. Hemorrhagic cyst was seen in almost half of them $46.3 \%(n=19)$. Patients in which ovarian torsion cannot be ruled out, who had a pain persisting longer than 48 hours, with a follow up for 1 or 2 menstrual cycles and had a cyst bigger then $5 \mathrm{~cm}$, and the patients who had pain symptoms were operated. None of the patients had malignant lesions.

Giant cysts, which are bigger than $15 \mathrm{~cm}$ in diameter, are commonly benign and functional. There may not be enough space for minimal invasive surgery in the abdomen in these cases. Drainage by ultrasonography, mini-laparotomy or laparoscopy guided aspiration is recommended (12). It is men- tioned that, these cysts are settled in pelvis so that torsion is not a possible risk for these patients $(12,13)$. In our cases, camera port was placed in epigastric area with Hasson technique and the working ports were placed in lateral sides of rectus abdominis muscle in upper abdomen. Veress or a suitable needle aspirated the cysts, percutaneously. After maintaining better exposure, cystectomy was performed. With this approach, cystic content was aspirated with camera control and the procedure was completed minimal invasively.

Ovarian torsion constitutes $2.7 \%$ of gynecological emergency situations. 14 Children commonly present with suddenly starting, continuous and non-specific abdominal pain $(3,4,7,14,15)$. Delayed diagnosis or misdiagnosis may cause organ damage or lost (14). Blood tests may reveal leukocytosis but there is not a correlation between leukocytosis and necrosis $(4,14)$. In our study, leukocytosis was found in $57 \%$ of the torsions. Ovarian torsion is rare among normal sized ovaries $(6,8)$. In the study of Oltmann et al., torsioned ovaries were over $5 \mathrm{~cm}$ in $83 \%$ of patients (16). But in the childhood, the long ovarian pedicle is the common underlying reason of torsion. Longer fallopian tube, mesosalpinx or mesoovarium provides mobility and makes torsion easier $(4,15,17,18)$ Torsion is rare among malignant pathologies and coincidental torsion rate is between $2-6 \%(2,7,17,18)$. It can be explained by adhesions to environmental tissues, which resulted from inflammation and fibrosis of malignancy (17-19) Left ovarian torsion is less common. It is thought that sigmoid colon prevents mobilization $(9,13,16,17,18)$ In our cases, it was concordant with the literature with a rate of $29 \%$ left ovarian torsion.

It is known that ultrasonography and color Doppler ultrasonography are useful for diagnosis $(2,9,14)$. Because of the edema, torsioned ovary may not be seen; or echogenic, hypoechoic mass or bigger ovary may be seen. Peripherally located folliculles are seen $(4,15,17,18,20)$. But the diagnosis rate is $13-50 \%$ with this view $(3,17)$ Absence of blood flow and whirlpool sign is susceptible for torsion. But due to the dual arterial blood flow, seeing the arterial blood flow does not exclude the torsion $(38-62 \%)(3,17,18,21)$ In the study with adult patients of Albayram et al., arterial and venous blood flow were not seen in $40 \%$ of the patients, venous blood flow was reduced and arterial blood flow was absent in 33\%, both arterial and venous blood flow were reduced in $13 \%$, arterial blood flow was reduced and venous blood flow were not seen in 7\% of the patients (22) In Ben - Ami et al.'s study, in 67\% of patients arterial and venous blood flow were not seen (23) In our study, increased size of ovary, cysts and peripherally located folliculles were stated. Color Doppler ultrasonography only revealed absence of arterial blood flow in four of the children $(28.5 \%)$. In one child, laparoscopy revealed torsion even though ultrasonography and color Doppler ultrasonography found no finding in favor of torsion. In one child with size increase and one with no blood flow in color Doppler ultrasonography, laparoscopy was performed but no pathology was 
identified. Magnetic resonance imaging and computed tomography can also be performed and expanded adnexia, peripheral cystic lesions, pelvic masses, vascular congestion signs, peritoneal fluid, tubal thickening and uterine deviation can sign torsion $(4,8,15,17)$ But in our clinic, computed tomography is not first choice due to the side effects. It is only performed in four children with radiologist's advice. Three cysts and one teratoma were revealed in these children, which were concordant with laparoscopy and histopathological diagnosis.

Ovaries are supplied in a dual arterial system. Because of this, venous system is affected first in the setting of torsion and congestion, edema, discolorization, ischemia and necrosis develop. Black-bluish color change, size and edema can be misleading. The situation is reversible because complete arterial obstruction does not generally happen $(4,8,14,17,24)$ Avoiding oopherectomy is accepted widely. Ovarian function return in $88-100 \% .8$ In the older papers, because of the tromboembolic risks, oopherectomy was suggested without detorsion when black bluish adnex was seen. But in the current studies, this ratio is reported to be no more than $0.02 \%$ $(3,4,15,17,25,26)$ and so that fertility can be protec $(2,5,7,17,18)$. Torsioned ovary is detorsioned. If secondary torsion develops, an attempt to the cyst must be made. This is necessary to get a histopathological sample and for avoidance of torsion.15 Without oopherectomy, malignancy can be overlooked $(17,26)$. In the case of identifying solid or heterogenic mass, frozen biopsy can be taken to rule out malignancy (17). After the detorsion cases without oopherectomy, follow-up of the ovary dimensions until it becomes normal or atrophic, is necessary to avoid overlooking of malignancies $(4,17)$. It is recommended to repeat the ultrasonography after 1-8 weeks and tumor markers if necessary $(4,17)$. In our routine, we repeat ultrasonography after one or five menstrual cycles. Oopherectomy was performed in four of sixteen torsion cases. Control ultrasonography examination revealed normal size and normal ovarian tissue in seven children among the others. In two children, residual ovarian tissue was seen and these children were followed for ovarian atrophy. Three children were out of follow up.

Though uncommon, ovarian torsions may repeat. The incidence is reported as $2-11 \%(18,27,28)$. Although most of the authors find it unnecessary, some of them advocate oophoropexy because of this $(3,4,8)$. In the case of ipsilateral or contralateral repeating, oophoropexy is widely accepted (17).

In the retrospective study of Spinelli et al, 137 lesions in 130 children are reported to be $46.7 \%$ functional lesion, $43.1 \%$ as benign neoplasm, $3.7 \%$ malign, $6.6 \%$ normal ovarian torsion. Oopherectomy was conducted in 56 children out of $130(40.9 \%)$ (2). In our study, oopherectomy was performed in $16.9 \%$ and protective approach took place whenever possible.

Definitive diagnosis is made surgically and histopatholog- ically in ovarian pathologies (17). In a study, pediatric surgeons and obstetricians were compared in 1151 patients less than 18 years of age diagnosed with ovarian torsion. Eightyone percent of the patients were intervened by pediatric surgeons. Twentyseven percent of pediatric surgeons managed laparoscopically compared to $17 \%$ by the obstetricians. All of our cases started laparoscopically but in one patient mini-laparotomy was performed. In the same study, $38 \%$ of the pediatric surgeons performed oopherectomy compared to $27 \%$ by the obstetricians (26). Dermoid cysts and teratoma was found in five of our cases. Oopherectomy was performed in only four cases, which was thought to be necrosis due to torsion. Our total oopherectomy rate was $16.9 \%$ but when the solid masses excluded, the rate reduces to $7.5 \%$.

Laparoscopic surgery is started to be used by the obstetricians earlier compared to pediatric surgeons but with the advance of the technology it is also widely accepted by pediatric surgeons (1) Diagnostic laparoscopy is the gold standard in children and it is also valuable in differential diagnosis. Laparoscopically performed conservative surgery is associated with less hospital stay, less blood loss, less postoperative pain, shorter recovery period, faster return to normal activity and superior cosmetic result. Compared to laparotomy, it is associated with less pelvic adhesions, which preserves fertility better.

\section{References}

1. Panteli C, Minocha A, Kulkarni MS, Tsang T. The role of laparoscopy in the management of adnexal lesions in children. Surg Laparosc Endosc Percutan Tech 2009; 19(6): 514-7.

2. Spinelli C, Pucci V, Strambi S, Piccolo RL, Martin A, Messineo A. Treatment of ovarian lesions in children and adolescents: a retrospective study of 130 cases. Pediatr Hematol Oncol 2015;32(3):199-206.

3. Geimanaite L, Trainavicius K. Ovarian torsion in children: management and outcomes. J Pediatr Surg 2013;48(9): 1946-53.

4. Bottomley C, Bourne T. Diagnosis and management of ovarian cyst accidents. Best Pract Res Clin Obstet Gynaecol 2009;23(5):711-24.

5. Akkoyun I, Gülen S. Laparoscopic cystectomy for the treatment of benign ovarian cysts in children: an analysis of 21cases. J Pediatr Adolesc Gynecol 2012;25(6):364-6.

6. Spinelli C, Di Giacomo M, Cei M, Mucci N. Functional ovarian lesions in children and adolescents: when to remove them. Gynecol Endocrinol 2009;25(5):294-8.

7. Broach AN, Mansuria SM, Sanfilppo JS. Pediatric and adolescent gynecologic laparoscopy. Clin Obstet Gynecol 2009;52(3):380-9.

8. Oelsner G, Shashar D. Adnexal torsion. Clin Obstet Gynecol 2006;49(3):459-63. 
9. Poonai N, Poonai C, Lim R, Lynch T. Pediatric ovarian torsion: case series and review of the literature. Can J Surg 2013;56(2):103-8.

10. Grabowski A, Korlacki W, Pasierbek M. Laparoscopy in elective and emergency management of ovarian pathology in children and adolescents. Wideochir Inne Tech Maloinwazyjne 2014;9(2):164-9.

11. Templeman C, Fallat ME, Blinchevsky A, Hertweck SP. Noninflammatory ovarian masses in girls and young women. Obstet Gynecol 2000;96(2):229-33.

12. Murawski M, Golebiewski A, Sroka M, Czuderna P. Laparoscopic management of giant ovarian cysts in adolescents. Wideochir Inne Tech Maloinwazyjne 2012;7(2):111-3.

13. Rousseau V, Massicot R, Darwish AA, et al. Emergency management and conservative surgery of ovarian torsion in children: a report of 40 cases. J Pediatr Adolesc Gynecol 2008;21(4):201-6.

14. Oelsner G, Cohen SB, Soriano D, Admon D, Mashiach S, Carp H. Minimal surgery for the twisted ischaemic adnexa can preserve ovarian function. Hum Reprod 2003;18(12):2599-602.

15. Kitporntheranunt M, Wong J, Siow A. Entangled bilateral adnexal torsion in a premenarchal girl: a laparoscopic approach. Singapore Med J 2011;52(6):e124-7.

16. Oltmann SC, Fischer A, Barber R, Huang R, Hicks B, Garcia N. Cannot exclude torsion a 15-year review. J Pediatr Surg 2009;44(6):1212-6.

17. Spinelli C, Buti I, Pucci V, et al. Adnexal torsion in children and adolescents: new trends to conservative surgical approach our experience and review of literature. Gynecol Endocrinol 2013;29(1):54-8.

18. Ashwal E, Krissi H, Hiersch L, Less S, Eitan R, Peled Y. Presentation, diagnosis, and treatment of ovarian torsion in premenarchal girls. J Pediatr Adolesc Gynecol 2015;28(6):526-9.
19. Savic D, Stankovic ZB, Djukic M, Mikovic Z, Djuricic S. Torsion of malignant ovarian tumors in childhood and adolescence. J Pediatr Endocrinol Metab 2008;21(11): 1073-8.

20. Kao JK, Chiu CC, Wang PY, Yu MK. Pediatric ovarian torsion in a medical center in Taiwan: case analysis. Pediatr Neonatol 2012;53(1):55-9.

21. Tsafrir Z, Hasson J, Levin I, Solomon E, Lessing JB, Azem F. Adnexal torsion: cystectomy and ovarian fixation are equally important in preventing recurrence. Eur J Obstet Gynecol Reprod Biol 2012;162(2):203-5.

22. Albayram F, Hamper UM. Ovarian and adnexal torsion: spectrum of sonographic findings with pathologic correlation. J Ultrasound Med 2001;20(10):1083-9.

23. Ben-Ami M, Perlitz Y, Haddad S. The effectiveness of spectral and color Doppler in predicting ovarian torsion. A prospective study. Eur J Obstet Gynecol Reprod Biol 2002;104(1):64-6.

24. Breech LL, Hillard PJ. Adnexal torsion in pediatric and adolescent girls. Curr Opin Obstet Gynecol 2005;17 (5):483-9.

25. McGovern PG, Noah R, Koenigsberg R, Little AB. Adnexal torsion and pulmonary embolism: case report and review of the literature. Obstet Gynecol Surv 1999; 54(9):601-8.

26. Campbell BT, Austin DM, Kahn O, et al. Current trends in the surgical treatment of pediatric ovarian torsion: we can do better. J Pediatr Surg 2015;50(8):1374-7.

27. Ozcan C, Celik A, Ozok G, Erdener A, Balik E. Adnexal torsion in children may have a catastrophic sequel: asynchronous bilateral torsion. J Pediatr Surg 2002;37(11): 1617-20.

28. Celik A, Ergün O, Aldemir H, et al. Long-term results of conservative management of adnexal torsion in children. $\mathrm{J}$ Pediatr Surg 2005;40(4):704-8. 